\title{
APLICATIVOS WEB EN EL DESARROLLO DE COMPETENCIAS EN INVESTIGACIÓN. UN MODELO
}

\section{WEB APPLICATIONS IN THE DEVELOPMENT OF RESEARCH COMPETENCES. A MODEL}

\author{
Mawency Vergel-Ortega ${ }^{1}$, \\ Luisa Stella paz Montes², \\ Carlos Sebastián Gómez-Vergel ${ }^{3}$ \\ UFPS
}

\section{RESUMEN}

La investigación tuvo como objetivo evaluar las estrategias de implementación de aplicativo web en programas académicos de pregrado, de facultades de administración e ingeniería. La investigación sigue un estudio nivel explicativo, diseño correlacional; la muestra la constituyen 500 estudiantes de instituciones educativas de la ciudad de Cúcuta. Resultados muestran que programa académico, género, procedencia, estrato, automatización no fueron variables asociadas al modelo

1 Departamento de Matemáticas y estadística, Universidad Francisco de Paula Santander, Colombia. Orcid: https://orcid.org/00000001-8285-2968 Correo electrónico: mawency@ ufps.edu.co

$2 \quad$ Universidad Francisco de Paula Santander, Colombia. Orcid: 0000-0002-8887-3441 Correo electrónico: luisastellapm@ufps.edu.co

3 Ingeniería Electrónica, Universidad de Los Andes, Colombia. Orcid: https://orcid.org/0000-0002-61763613 Correo electrónico: cs.gomezv@uniandes.edu.co predictor, los recursos y servicios virtuales diseñados e implementados aportaron a la promoción de prácticas de aplicación asociadas a generación de algoritmos y competencias en procesos lógicos en la solución de problemas, procesos metalingüísticos, creatividad tiene alta asociación a investigación. Se concluye que el desarrollo de competencias en investigación se relaciona con variables predictores de perfil del estudiante en su formación previa en educación media, desarrollo del pensamiento lógico, a competencias en metodología e investigación en su formación profesional, así como la orientación al desarrollo de algoritmos.

\section{PALABRAS CLAVES:}

plataforma digital, educación superior, competencias, tic. 


\section{ABSTRACT}

The objective of the research was to evaluate the implementation strategies of web applications in undergraduate academic programs of administration and engineering faculties. The research follows an explanatory level study, correlational design; the sample is constituted by 500 students from educational institutions in the city of Cúcuta. Results show that academic program, gender, origin, stratum, automation were not variables associated to the predictor model, the resources and virtual services designed and implemented contributed to the promotion of application practices associated to the generation of algorithms and competences in logical processes in the solution of problems, metalinguistic processes, creativity has a high association with research. It is concluded that the development of research competencies is related to predictor variables of the student's profile in their previous training in secondary education, development of logical thinking, methodology and research topics incorporated in each module of the curricular program, as well as the orientation to the development of algorithms.

\section{KEYWORDS:}

digital writing center, middle education, academic writing.

\section{INTRODUCCIÓN}

El desarrollo de competencias en educación superior, han sido abordados desde diversas perspectivas, ya sea desde la asesoría [1], individual presencial, alfabetización [2], talleres [3], prácticas reales, la lectura crítica como práctica sociocultural [4], proyectos dirigidos a brindar soluciones a problemáticas de índole social, económica, política, de género, educativo, ingenieril u otro. Sin embargo, se busca incorporar herramientas didácticas que potencien la actividad fuera del aula, máxime esfuerzos hacia la investigación, donde la tecnología está al alcance de jóvenes, para además mejorar la lectoescritura en todos los niveles y áreas del currículo universitario en pregrado. Transformaciones en la dimensión comunicativa de los estudiantes de instituciones educativas fueron asociadas a la realidad de los sujetos participantes [5], en la medida en que incorpora escenarios reales [6. Generar competencias investigativas y de innovación, está relacionado con un proceso cognoscitivo [7], por cuanto el estudiante de pregrado debe ser capaz de elaborar conceptos a partir de la observación de las realidades existentes en el contexto y el estudiante de postgrado debe crear, generar nuevo conocimiento [8], conformar micro-estructuras de comunidad como actores activos del proceso [3], aborden epistemológica y metodológicamente sus investigaciones, utilicen competencias de lecto-escritura crítica para comunicarse, participar en redes, compartir avances científicos difundidos por redes, instituciones, profesores y semilleros; y quienes son maestros incorporen didácticamente esas posibilidades comunicativas y sociales para lograr desarrollo de habilidades del pensamiento y procesos de aprendizaje [9], así mismo desde el acompañamiento didáctico permanente, la intervención de prácticas de lecto-escritura [10], y desde una cultura digital [11] como proceso de composición textual soportado en recursos electrónicos que genera transformaciones en la producción [12], el procesamiento y la transmisión de la información [13]. Dada la creación de aplicativos web y herramientas como WhatsApp por parte de profesores y de la, herramientas que buscan promover prácticas de escritura o posibilidad para el aprendizaje de ciencias.

Se analiza el impacto de aplicativos web de la Universidad Francisco de Paula Santander, en programas de administración e ingeniería, y apoyo de chat de WhatsApp, correo; se determinan variables intervinientes y a partir 
de análisis multinivel, se identifican niveles de análisis a partir de variables personales y académicas, su asociación, relación o incidencia a competencias de innovación en los estudiantes. Las estrategias de implementación se diseñaron y operaron en función del objeto de estudio se asume desde las relaciones entre pensamiento [14], rendimiento, innovación en la construcción de conocimiento y difusión del conocimiento.

\section{METODOLOGÍA}

La presente investigación sigue un enfoque cuantitativo, nivel explicativo, de campo, cuasi experimental y transversal [15], con apoyo de análisis cualitativo; se realizó durante I-2019 a I-2021, la muestra fue conformada por 500 estudiantes de pregrado que cursaban de primer a cuarto semestre en programas de administración e ingeniería. La primera fase inicial implicó reconocimiento de contexto, servicio biblioteca virtual, currículo, aplicativos web existentes en la enseñanza del cálculo y software de apoyo en programas para crear aplicativos, documentos, tareas, material audiovisual, seguimiento. Variables predictoras se toman del modelo multinivel para postgrado e ingeniería en semestres superiores a cuarto, donde las variables son género, edad, procedencia, título en educación media (bachiller académico, bachiller técnico), estudio de pregrado que cursa, grupo o semillero de investigación, movilidad, productos de investigación, desarrollo pensamiento lógico, competencia metodológica y de investigación, teórica, lecto-escritura crítica, libertad de aprendizaje, valores y actitudes). Se diseñó un cuestionario estructurado para identificar aspectos personales y académicos de estudiantes de pregrado y maestría, contentiva de 10 preguntas de selección múltiple, mutuamente excluyentes [16], y 20 ítems en escala Likert de cinco opciones de respuestas para conocer las competencias investigativas, la segunda fase corresponde a análisis previo de resultados [16], seguimiento y validez de estrategias de implementación, se evalúa instrumento para plad con alfa de Cronbach [17] desarrollo pensamiento lógico 0.95 fiabilidad alta; competencia metodológica e investigativa 0.95 fiabilidad muy alta; teórica 0.91 fiabilidad alta, procesos metalingüísticos 0.92 muy alta; creatividad e innovación 0.94 fiabilidad muy alta, valor y actitud 0.8 alta; competencias libertad de aprendizaje 0.91 muy alta; se evalúa instrumento para WhatsApp con alfa de Cronbach desarrollo del pensamiento lógico 0.92 , fiabilidad alta; competencia metodológica e investigativa 0.92 fiabilidad muy alta; teórica 0.9 fiabilidad alta, técnica 0.8 fiabilidad alta, metalingüísticos 0.9 muy alta; creativa e innovación 0.94 fiabilidad muy alta, valor y actitud 0.85 alta; competencias libertad de aprendizaje 0.95 alta.

Promedios centrados de las dimensiones de la variable competencias innovación se catalogan en un nivel dos al seguir un modelo multinivel [18], se centra la variable predictora

$$
x_{i i}=X_{i}-X,[19] \quad \text { cambia el valor de la }
$$
intersección, pero no el valor de la pendiente ni de residuales; $X_{i}$ es el valor de la variable independiente original obtenida del estudiante $\mathrm{i}$ y $X$ es el valor promedio de pregrado o maestría para esa variable. La variable dependiente $Y_{i}$ se obtuvo mediante la sumatoria de los puntajes obtenidos en cuestionario en cada una de las dimensiones $X_{i}, Y_{i}=\sum_{i=1}^{8} X i$, se analiza variabilidad a partir de análisis de varianza ANOVA, ajustes de modelos e interacción por nivel [18]. Los efectos aleatorios asociados al nivel pregrado y maestría, se distribuyen normalmente, $e_{i j} \sim N\left(0, \sigma^{2}\right)$ y $u_{0 j} \sim N\left(0, \sigma_{u o}^{2}\right)$ [19].

Se analiza normalidad y homogeneidad a partir de estadísticos y estimaciones de parámetros aleatorios. Se evaluó la calidad del modelo propuesto a través del Coeficiente de determinación $R^{2}$ [16], teniendo en cuenta varianza explicada por el modelo. 


\section{RESULTADOS Y DISCUSIÓN}

Análisis previo cualitativo permite observar que el chat es el medio más utilizado, el uso de las plataformas y herramientas no tuvo diferencia significativa en estudiantes de pregrado y postgrado, tutoría desde la plataforma o whatsApp utilizó comunicación sincrónica, tutor y estudiante conectados con solicitudes o explicaciones breves por parte del estudiante y orientaciones de profesores utilizando cámara, redactando por voz, o escribiendo; no obstante, en las instituciones, la tutoría virtual fue poco explorada, los estudiantes preferían asesoría presencial [20], y chat fue más utilizado para preguntas concretas de experimentos 0 realización de prototipos. La tutoría académica sigue siendo el factor diferenciador de la plataforma digital [14]. La posibilidad de orientar y acompañar a los estudiantes en su proceso por parte de un tutor [21] se convirtió en una oportunidad de diálogo desde fortalezas y debilidades que requerían, de momentos de planeación, diálogo, revisión y realización de adaptaciones didácticas continuas, así como de apoyo de otros profesionales de otras áreas para resolver dudas como profesores frente a dudas de estudiantes o planteamiento de nuevos retos aplicados por parte de los mismos.

Basado en el modelo multinivel para postgrado y programas de ingeniería para estudiante de semestres superiores a cuarto [16], el análisis cuantitativo permite analizar variables predictores que no presentan correlación (movilidad, género, edad, pregrado, semestre, uso chat, uso biblioteca), moderada (teórico, valor y actitud), alta (procesos metalingüísticos 0.79; competencia metodológica e investigativa 0.84; desarrollo pensamiento lógico 0. 91, competencias libertad de aprendizaje 0.8) correlación positiva con la variable dependiente; creatividad e innovación, siendo las variables formación $\rho_{x, y}=0.92$, competencia metodológica e investigativa (MI) con $\rho_{x, y}=0.91$, desarrollo del pensamiento lógico ( $\mathrm{L}$ ) con

$\rho_{x, y}=0.91$, procesos metalingüísticos

$\rho_{x, y}=0.83$ y libertad de aprendizaje $\rho_{x, y}=0.8$, las de mayor correlación con la variable dependiente a un nivel de significación del $5 \%$. Se selecciona la variable formación $(F)$, por tener el mayor coeficiente de correlación con la variable dependiente competencias innovación $\rho_{x, y}=0.95$.

Se toma como base modelo lineal lineal $y_{i j}$, i-ésimo estudiante j-ésimo programa. Se incorporaron covariables del nivel 1 resultado de análisis cualitativo al modelo, surgiendo así el modelo el modelo incondicional para pregrado $y_{i j}$ y covariables de segundo nivel $\beta_{0 j}$ [16], respectivamente con :

$$
\begin{gathered}
y_{i j}=\beta_{0 j}+e_{i j} \\
\beta_{0 j}=F_{00}+F_{01} L_{1 j}+M I_{02} L I N_{2 j}+u_{o j} \\
\operatorname{con} L_{j}=L_{j}-\bar{L}
\end{gathered}
$$

$F_{00}$ representa las competencias en ciencias promedio global de los estudiantes, intercepto o media total de la variable innovación; $u_{o j}$ es la variabilidad que existe entre los programas en términos de las competencias de innovación promedio de los estudiantes; $e_{i j}$ denota la variabilidad que existe en las competencias de innovación de los estudiantes anidados en el j-ésima programa académico, $F_{01}$ y $F_{02}$ son las pendientes en regresión que acompañan a las variables independientes fijas del nivel 2. para construir el modelo lineal multinivel se seleccionan las variables del nivel 3: automatización (A) con $\rho_{x, y}=0.91$, algoritmización y riesgo financiero (AF) denominada digitalización de la economía en postgrado con $\rho_{x, y}=0.95 \mathrm{y}$, del nivel 2 lógico_C $\left(\mathrm{L} \_\mathrm{C}\right)$, reconocimiento de imágenes para la toma 
de decisiones y metalingüístico_C (LIN_C), .

$u_{0 j} \sim N\left(0, \sigma_{u 0}^{2}\right), u_{p j} \sim N\left(0, \sigma_{u p}^{2}\right), e_{i j} \sim N\left(0, \sigma^{2}\right)$.

Nivel 1:

$$
y_{i j}=\beta_{o j}+\beta_{1 j} x_{1 i j}+\beta_{2 j} x_{2 i j}+\beta_{3 j} x_{3 i j}+e_{i j}
$$

Nivel 2: Intercepto:

$$
\beta_{0 j}=F_{00}+F_{01} L_{1 j}+M I_{02} L_{I N} N_{2 j}+u_{0 j}
$$

Pendientes:

$$
\beta_{p j}=\sum_{p=1}^{3} F_{p 0}+\sum_{p=1}^{3} \sum_{q=1}^{2} M I_{p q} L_{q j}+\sum_{p=1}^{P} u_{p j}
$$

De donde

$$
y_{i j}=F_{o o}+\sum_{q=1}^{2} F_{0 q} L_{q j}+\sum_{p=1}^{3} F_{p 0} x_{p i j}
$$

$+\sum_{p=1}^{3} \sum_{q=1}^{2} M I_{p q} L_{q j} x_{p i j}+u_{0 j}+\sum_{p=1}^{3} u_{p j} x_{p i j}+e_{i j}$

Para verificar que la incorporación de dichos términos de error es significativa se aplica un Test de $\mathrm{X}^{2}$ de Verosimilitud tomando como referencia al modelo $\mathrm{Cl}$, como se observa en la Tabla 1 la inclusión de las variables $\mathrm{A}, \mathrm{AF}, \mathrm{MI}$, como términos de error al modelo $\mathrm{Cl}$ demuestra que son significativas ya que el p-valor resultó ser

$$
\begin{aligned}
& C I_{i j}=F_{00}+F_{01} L_{-} C_{j}+M I_{02} L I N_{-} C_{j}+M I_{10} A_{i j} \\
& +M I_{20} L_{i j}+M I_{30} L I N e_{i j}+F_{11} L_{-} C_{j} * A_{i j}+F_{12} L I N e_{C_{j}} \\
& * A_{i j}+F_{21} L_{-} C_{j} * L_{i j}+F_{22} L I N e_{-} C_{j} * L_{i j}+ \\
& F_{31} L_{-} C_{j} * L I N e_{i j}+F_{32} L I N e_{-} C_{j} * L I N e_{i j} \\
& +u_{o j}+u_{1 j} A F_{i j}+u_{2 j} L_{i j}+u_{1 j} L I N e_{i j}+e_{i j}
\end{aligned}
$$

Al realizar test de contrastes de efectos fijos para los modelos de componentes de varianzas definidos para las competencias de innovación, se genera el Modelo Cl:

$$
C I_{i j}=F_{00}+F_{01} L_{-} C_{j}+M I_{02} L I N_{-} C_{j}+M I_{10} A_{i j}+
$$

$M I_{20} L_{i j}+M I_{30} L I N e_{i j}+F_{11} L_{-} C_{j} * A_{i j}+u_{o j}+u_{1 j} A F_{i j}$

$+u_{2 j} L_{i j}+u_{1 j} L I N e_{i j}+e_{i j}$

menor a 0.04; por consiguiente, las pendientes medias que relacionan las competencias investigativas con las puntuaciones de las variables del nivel 1 son significativas, por lo que estas variables predictores pueden ser seleccionadas como parte aleatoria del modelo Cl.

Tabla 1. Test de $\mathrm{X}^{2}$ de Verosimilitud para los modelos obtenidos al incluir el efecto aleatorio para cada variable predictora de las competencias investigativas de los estudiantes

\begin{tabular}{|l|c|c|c|c|c|}
\hline \multirow{2}{*}{ Variable } & $\begin{array}{c}\text { Modelo reducido } \\
\text { 1 parámetro }\end{array}$ & $\begin{array}{c}\text { Modelo completo } \\
\text { (con variable incluida 2 } \\
\text { parámetros) }\end{array}$ & Deviance & gl & p-valor \\
\cline { 2 - 4 } & $\mathbf{- 2 L L}$ & $-\mathbf{2 L L}$ & 8,015 & 1 & 0,0035 \\
\hline $\mathrm{A}$ & 1235,802 & 1415,67 & 9,057 & 1 & 0,0003 \\
\hline $\mathrm{AF}$ & 1235,807 & 1417,69 & 9,521 & 1 & 0,0034 \\
\hline
\end{tabular}

Fuente: elaboración propia 
De acuerdo con la ecuación 8, para la selección de las variables predictores incluidas en el modelo lineal multinivel definitivo se procedió a analizar parámetros, donde se usó una estructura de covarianzas de tipo identidad Tabla 2.

Tabla 2. Estimación de los parámetros fijos del Modelo

\begin{tabular}{|l|c|c|c|c|}
\hline \multicolumn{1}{|c|}{ Parámetro } & Estimación & Error típico (SE) & & p-valor \\
\hline Intersección & 7,7150 & 9,8393 & 0,650 & 0,515 \\
\hline A & 0,5316 & 2,4935 & 0,293 & 0,879 \\
\hline L & 3,0756 & 0,2730 & 8,739 & 0,001 \\
\hline MI_C & 0,7418 & 0,5813 & 1,257 & 0,257 \\
\hline L_C & $-0,4266$ & 0,2632 & $-1,506$ & 0,151 \\
\hline LIN_C & 1,2922 & 0,6058 & 2,133 & 0,056 \\
\hline A*L_C & 7,2506 & 9,1005 & 9,847 & 0,275 \\
\hline
\end{tabular}

Fuente: elaboración propia

De los resultados se observa que solo las variables competencias de desarrollo de pensamiento lógico, metodología e investigativo resultaron ser significativas al 5\%; tanto el intercepto como las variables: $\mathrm{A}, \mathrm{MI}, \mathrm{L} \_\mathrm{C}$ y la interacción $A^{*} L_{-} C$ se excluyen del modelo $\mathrm{Cl}$ por no ser significativas. El modelo $\mathrm{Cl}$ tiene la estructura:

$$
C I_{i j}=F_{01} M I_{-} C_{j}+F_{10} L_{i j}-M I_{11}+u_{o j}+e_{i j}
$$

Donde $F_{10}$ indica el cambio en las Cl promedio de los estudiantes por cada unidad de aumento en nivel de desarrollo de pensamiento lógico $L$ controlando los efectos de las variables restantes, o la relación existente entre las $\mathrm{L}$ y las $\mathrm{Cl}$, controlando los efectos de las variables restantes; $M I_{11}$ indica el cambio en las competencias investigativas promedios de los estudiantes como consecuencia de las competencias de desarrollo del pensamiento lógico y; $F_{01}$ es la interacción cruzada entre competencias propias de la formación en educación básica y las competencias metodológica investigativa centrada; $u_{o j}$ es el efecto aleatorio del $j$-ésima programa que cursa sobre las medias $\mathrm{y} ; e_{i j}$ es el error aleatorio del nivel 1, asumido con distribución normal e independiente con media cero y varianzas constantes $\left(\sigma_{u 0}^{2}, \sigma_{e}^{2}\right)$ respectivamente.

En el modelo $\mathrm{Cl}$ se procedió a realizar un análisis excluyendo la variable $L$ del componente aleatorio (modelo reducido) e incluyendo dicha variable en el componente aleatorio (modelo completo), cuyos resultados muestran parámetros fijos en modelo reducido $L 2.5^{\star * *}$ (0.01), MI_C $1.05^{\star * *}(0.15)$, y aleatorios $\widehat{\sigma}_{\mathbf{u} 0}^{2}=21$ promedio (16.15), residuos : $\widehat{\boldsymbol{\sigma}}_{\mathbf{e}}^{2} 45^{\star * *}$ (4.5) -2 log de la verosimilitud restringida $1.455,046 ;{ }^{* * *} \mathrm{p}<0.01 ; y$ en modelo completo reducido L 2.1 (7.01), MI_C $1^{* * *}(0.15)$. Al comparar el estadístico -2LL entre ambos modelos resultó ser menor el del modelo reducido; aunque en ambos casos son significativos al compáralo con una distribución $\mathrm{X}^{2}$. Por lo 
tanto, el modelo final queda estructurado de la siguiente manera:

$$
\begin{aligned}
& C I_{i j}=1,05 * F_{01}+2,5 * F_{10}-M I_{11}+u_{o j}+e_{i j} \\
& \hat{\sigma}_{e}^{2}=40 \quad \hat{\sigma}_{u o}^{2}=20.5
\end{aligned}
$$

El coeficiente de determinación $\mathrm{R}^{2}$ para el nivel de estudiantes es 0.95 con el que se estaría explicando el $95 \%$ de la variabilidad observada en las competencias innovación. Por lo tanto, el modelo $\mathrm{Cl}$ es adecuado para describir la relación existente entre las variables predictores seleccionadas con las competencias de innovación de los estudiantes de administración e ingeniería.

\section{CONCLUSIONES}

Estrategias de implementación para el fortalecimiento de la investigación en programas académicos de administración e ingeniería está asociado a competencia adquirida en educación básica y media, a competencias en metodología e investigación en su formación profesional, al desarrollo del pensamiento lógico, y el desarrollo de algoritmos.

\section{AGRADECIMIENTOS}

A los Grupos de investigación Euler en Educación matemática, conocimiento e innovación social Quetelt y Graunt en Estadística aplicada y al Fondo de Investigación universitarias FINU de la Universidad Francisco de Paula Santander (Colombia).

\section{REFERENCIAS}

[1] N. Bernal, M. Muñoz, and M. Vergel, "Fomento de la convivencia en instituciones de educación superior desde la Consejería académica y de investigación," Revista Logos, ciencia y tecnología, vol. 10, no. 1 , p. 69,2018 , doi: 10.22335/rlct. v10i1.517

[2] J. Cabero Almenara, y M. Llorent, "La alfabetización digital de los alumnos, competencias digitales para el siglo XXI," Revista portuguesa de pedagogía, vol 42 no. 2, p. 7-28, 2008.

[3] P. Carlino, "Alfabetización académica diez años después," Revista Mexicana de Investigación Educativa, vol. 38, no. 57, pp. 355-381, 2013.

[4] B. Figueroa, M. Aillon y A. Fuentealba. "La escritura académica con soporte de esquemas digitales en la formación docente". Revista de Universidad y Sociedad del Conocimiento (RUSC). vol. 11 no. 1, 18-32, 2014 doi: $\underline{10.7238 /}$ rusc.v11i1.1665

[5] Rolls-Royce plc, The jet engine., 5th ed. Wiley, 2015.

[6] A. Ortega, M. Vergel y J. Martinez, "Validity of microrubri, instrument to measure the development of competences in mathematics Journal of Physics: Conference Series 1160 p. 125.

[7] H. Parra-López, J. Rojas-Suarez and M. Vergel-Ortega, "Curricular trends in the Universidad Francisco de Paula Santander academic program offerings," Journal of Physics: conferences series vol. 1329 no. 3201 p. 9, 2019.

[8] M. Vergel, J. Martínez and S. Zafra, "Apps en el rendimiento académico y auto 
concepto de estudiantes de ingeniería", Revista Logos Ciencia \& Tecnología vol. 6 no. 1, p. 98, 2015.

[9] M. Fujioka, "U.S. writing center theory and practice: implications for writing centers in Japanese universities", Kinki University Center for Liberal Arts and Foreign Languages Education Journal, vol. 2 no. 1, pp. $205-224,2011$.

[10] K. Guzmán Tinajero y S. Rojas-Drummond, "Escritura colaborativa en alumnos de primaria: un modelo social de aprender juntos," Revista mexicana de investigación educativa, vol. 17, no. 52, pp. 217-245, 2012.

[11] G. Hays, "Learners helping learners in an EFL writing center", JALT2009 Conference Proceedings, vol. 17 no. 1 , 2014.

[12] A. J. Stepanoff, Centrifugal and axial flow pumps. Theory, design and application. Wiley, 1957

[13] B. Tan, "Innovating writing centers and online writing labs outside North America," The Asian EFL Journal Quarterly, vol. 13, no. 2, pp. 390-417, 2015.

[14] S. Vivant, "Digital technology as a tool for the democratization of powerful mathematical ideas," Revista Colombiana de Educación, vol. 74 no. 1, p. 10, 2018.

[15] J. Nieto, J. Rojas and M. Vergel. Impacto de estrategia pedagógica basada en el aprendizaje creativo para estudiantes de ingeniería. Editorial Ibáñez, 2019

[16] M. Vergel-Ortega, Z. C. Nieto-Sánchez, C. S. Gómez-Vergel, 2021. Predictores de innovación en programas de ingeniería $y$ postgrado utilizando estrategias basadas en plataformas digitales," Rev. UIS Ing., vol. 20, no. 1, pp.213-222, doi: 10.18273/revuin.v20n1-2021018.

[17] A. Pineda Repizzo. Una cultura de la tutoría: Fundamentación de un sistema de tutorías desde el programa Taller de Lenguaje de la FUAC, Pontificia Universidad Javeriana, 2015.

[18] F. Murillo, "Los modelos multinivel como herramienta para la investigación educativa," Revista Internacional de Investigación en Educación, vol. 1, pp. 45-62, 2008.

[19] C. Werner, "Constructing Student Learning through Faculty Development: Writing Experts, Writing Centers, and Faculty Resources" CEA Forum, College English Association, vol. 42, no. 2, pp. 79-92, 2013.

[20] M. Vergel-Ortega, J. Martínez y S. Zafra, "Validez de instrumento para medir la calidad de vida en la juventud: VIHDA," Revista Logos Ciencia \& Tecnología vol. 7, no. 20, 2015.

[21] J. Martínez, M. Vergel y S. Zafra. Comportamiento juvenil y competencias pro-sociales. Editorial Ibáñez, 2016. 